\title{
Design and Evaluation of a Smart Library using the APEX Framework
}

\author{
Tiago Abade ${ }^{1,2}$, Tiago Gomes ${ }^{1,2}$, José Luís Silva ${ }^{3}$, and José C. Campos ${ }^{1,2}$ \\ 1 Departamento de Informática, Universidade do Minho, Braga, Portugal \\ 2 HASLab / INESC TEC, Braga, Portugal \\ 3 Madeira-ITI/Universidade da Madeira, Funchal, Portugal \\ \{pg20691@alunos.uminho.pt, pg19814@alunos.uminho.pt, \\ jose.1.silva@m-iti.org, jose.campos@di.uminho.pt\}
}

\begin{abstract}
User experience is a key point for successful ubiquitous computing (ubicomp) environments. The envisaged design should be explored as soon as possible to anticipate potential user problems, thus reducing re-design costs. The development of ubicomp environments' prototypes might help, providing feedback on the users' reaction to the environments. This paper describes the design and evaluation of ubicomp environments using APEX, a rapid prototyping framework providing user experience via a 3D application server and connected physical devices. APEX prototypes allow users to explore and experience many characteristics of a proposed design, in a virtual world. The paper focus in particular the design and evaluation of a smart library in the APEX framework.
\end{abstract}

Keywords: ubiquitous computing, 3D environments, prototyping, evaluation

\section{Introduction}

The design and engineering of interactive systems presents several challenges. The presence of a human factor means that the impact of design decisions is hard to predict and assess without being tested with actual users. This assessment, however, must be done as early as possible, before too many resources have been invested and too much time elapsed. Even though tools have been proposed that automate specific aspects of interactive systems' analysis [2,9], the use of prototypes remains the main approach to support early evaluation through user testing. Prototypes allow a system to be explored and/or analysed at an early stage avoiding the costs of producing a complete enough system to be ready for user testing.

Ubicomp environments pose specific challenges in terms of prototyping. Due to their physical and spatial nature, prototypes to enable an assessement of the experience of using (being in) those systems can become costly to develop and deploy. The acquisition of adequate resources (e.g. physical devices, sensors), deployment to its intended target location, and subsequent experimentation,

Preprint of the paper published in Distributed, Ambient, and Pervasive Interactions, volume 8530 of Lecture Notes in Computer Science, 2014. The final publication is available at Springer via: http://dx.doi.org/10.1007/978-3-319-07788-8_29. 
all have expensive associated costs. Design decisions, once committed to, can be difficult to reverse [4]. In some cases, fielding a system for the purpose of testing might not be possible. Prototypes based on simulations in 3D virtual environments provide designers with a way of checking proposed design solutions with relatively low investment.

This paper illustrates the use of APEX [17,18] which is a framework for rapid prototyping of ubicomp environments. The framework reduces the costs of development by providing, among other features, the means to experience the system before physical deployment, based on the 3D environment delivered. The framework is being used to demonstrate the value of the approach for early evaluation of ubiquitous environments including aspects of user experience before physical deployment in situ. The paper describes the approach and illustrates it through the design and evaluation of the prototype of a smart library. The evaluation aims to check and understand whether the envisaged design suits the goals and correctly adapts to the needs and expectations of users.

The paper is structured as follow: Section 2 presents related work and describes the APEX platform. Section 3 presents the case study used. The prototype is presented in Section 4. Section 5 describes a user study made and Section 6 summarised with conclusions and future work.

\section{APEX framework and related work}

Although several approaches that aim to prototype ubicomp environments can be identified (3DSim [11], UbiWorld [3], the work of O'Neill et al. [13], and VARU [7] are examples), they are mainly focused on helping ubiquitous system designers to identify unwanted behaviour in their systems, and to support informed decision making in an iterative design cycle. Other approaches (UbiWise [1], UbiREAL [12], d.tools [6] or Topiary [10]) focus on prototyping ubicomp applications or isolated devices and not on the prototyping of ubicomp environments as a whole. Some approaches allow an exploration of user experience but not of the whole ubicomp environment. Silva [14] provides an in-depth overview of the various approaches.

The absence of an approach that focuses on the experience that users will have of the design of the whole ubicomp environment, and which supports a formal and exhaustive analysis is remedied by APEX. The APEX framework is described in detail in $[14,18]$. Here, we provide a brief outline of its main characteristics.

The framework brings together an existing 3D Application Server (OpenSimulator $\left.^{4}\right)$ with a modelling tool $\left(\mathrm{CPN}\right.$ Tools $\left.{ }^{5}\right)$. APEX-based prototypes enable users to navigate and interact with a virtual world simulation of the envisaged ubiquitous environment. By this means users can experience many of the features of the proposed design. Prototypes are generated in the framework to help

\footnotetext{
${ }^{4}$ http://opensimulator.org (accessed: 6 December 2013)

${ }^{5}$ http://cpntools.org/ (accessed: 6 December 2013)
} 
the developer understand how the user might experience the system. The two main features of APEX are:

- Allowing the rapid prototyping of ubicomp environments;

- Providing a 3D virtual environment as a basis to represent the system to be developed that can be explored by users in a realistic way.

3D application servers, such as SecondLife ${ }^{6}$ or OpenSimulator provide a fast means of developing virtual worlds. OpenSimulator in particularly, has the advantage of providing access to the source code, thus supporting extensibility and better configurability.

APEX uses the formal notation, Coloured Petri Nets (CPN) [8], to describe the behaviour of the virtual environment. This behavioural model drives the virtual environment running OpenSimulator. This process is achieved using a specially designed APEX component. Using a combination of purpose built components[5], object warehouses, and an appropriate off-the-shelf viewer (e.g. Cool VL viewer ${ }^{7}$ ) APEX supports the construction of interactive and rich environments, providing users with an experience close to that of being in the real environment. Several users can establish connections simultaneously using different points of view in the OpenSimulator server. The users experience the proposed solution by navigating and interacting with the simulation as an avatar controled by mouse/keyboard, wiimote or smartphone.

The overall architectural view of the framework is presented in Figure 1. The five main components are:

1. a behavioural component, responsible for managing the behaviour of the prototype, including the description, analysis and validation of the virtual environment's behaviour;

2. a virtual environment component, responsible for managing the physical appearance and layout of the prototype, including managing the $3 \mathrm{D}$ simulation and the construction of the virtual environment;

3. a physical component, responsible for supporting connections to physical external devices, such as smart phones and sensors;

4. a communication/execution component, responsible for the data exchange among all components and for the execution of the simulation;

5. a configuration tool component, responsible for supporting the setting up and management of the framework.

These components support an approach to prototyping based on layers: Simulation layer, using OpenSimulator; Modelling layer, using the CPN models; Physical layer, using external devices and real data.

Developers can choose the level of abstraction they need to understand the design while prototyping the environment. APEX allows developers to switch between layers to evaluate different characteristics of the prototype. These characteristics might include the experience of the user using a physical device, or

\footnotetext{
${ }^{6} \mathrm{http}: / /$ secondlife.com/ (accessed: 6 December 2013)

7 http://sldev.free.fr/ (accessed: 6 December 2013)
} 


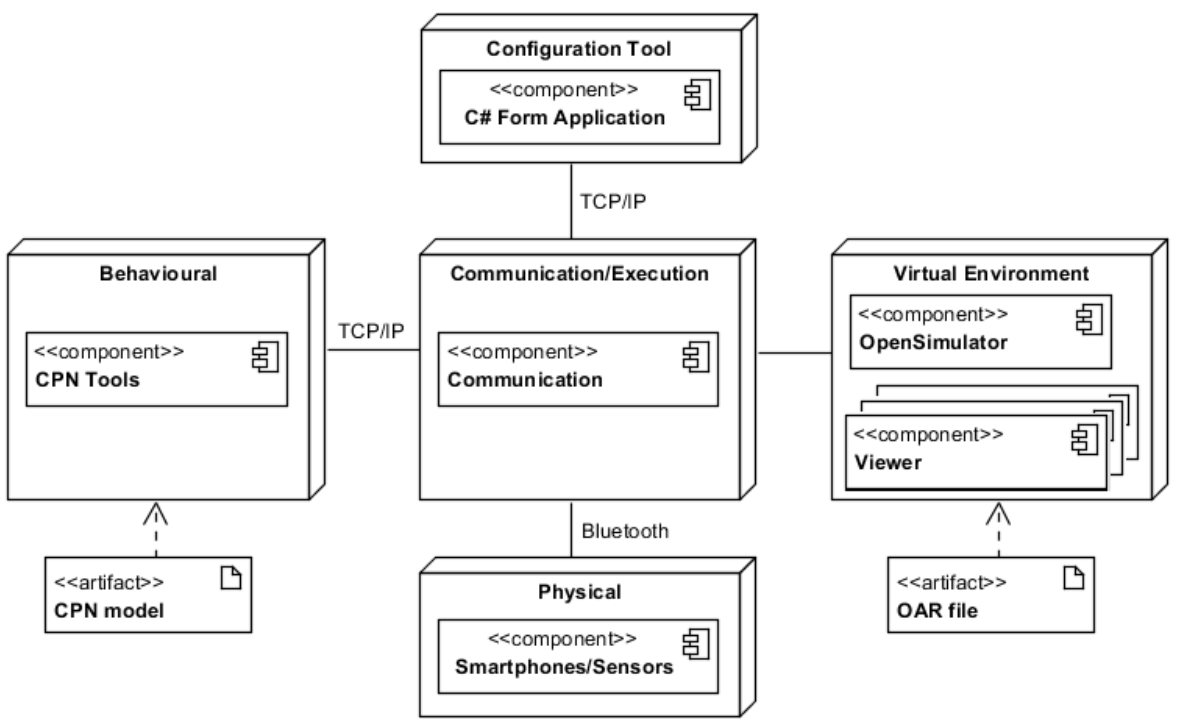

Fig. 1. APEX architecture

using the physical layer or alternatively providing an analysis of the behaviour of the system using the Modelling layer. For further information about the APEX framework, see $[15,17,16,14,18]$.

\section{Prototyping a smart library}

The introduction of ubiquitous computing technology into an existing library was explored using the facilities provided by APEX. The prototype included displays to provide information about the availability of seats, and sensors to determine which seats are occupied. The proposed design was assessed through user tests. The costs involved in deploying the solution to the actual library, including the potential for disruption, means that performing such tests on the actual system was not possible.

\subsection{The library}

The system is based on a specific library at the University of Minho, located in the Gualtar campus in Braga (Portugal). The building has 3 floors, including a ground-floor where the main reception is located. The other two floors are identical and have reading and study rooms (see Figure 2). Each of these two floors is composed of a reception (on the right in the figure) surrounded by doors that give access to the reading/study rooms. These rooms have 6 table sections (marked with circles in Figure 2). 


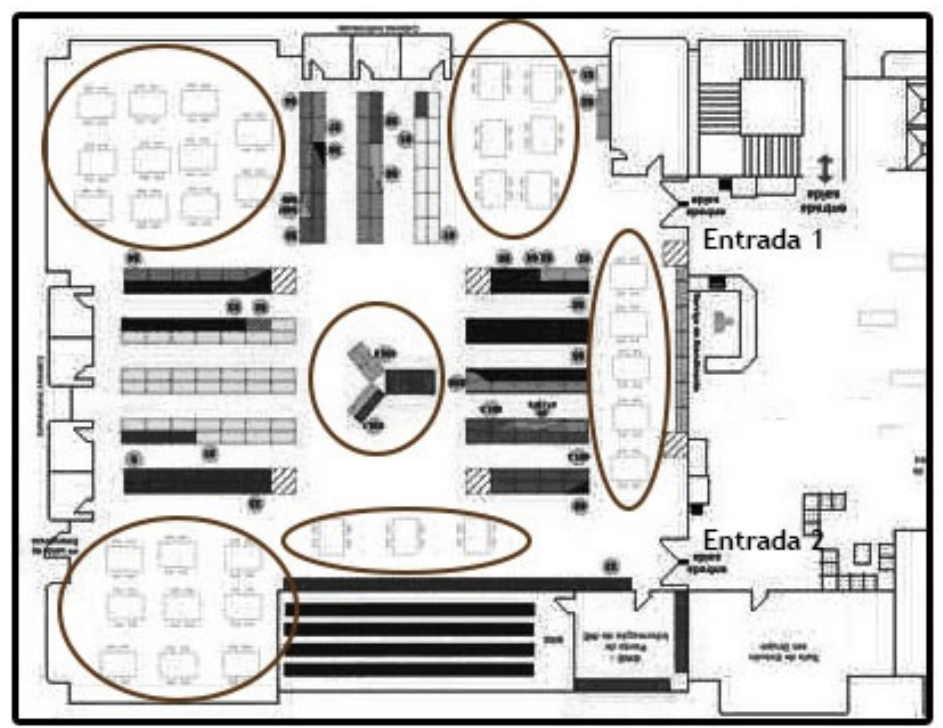

Fig. 2. Table sections of the Gualtar's library

The library is equipped with authentication systems for books and material requests. It does not currently provide any counting system to keep track of seat availability. The proposed enhancement of the library with ubicomp technology enables indication of seat availability. It is designed to illustrate the APEX approach.

\section{The prototype}

A 3D model of the library was built using OpenSimulator. The virtual environment was then enriched by introducing simulated technological solutions (sensors and public displays) to help users to identify available seats. CPN models that drive the ubicomp environment were added to support the additional services provided by the library.

Two types of information panels were simulated to provide information about seats in the library. The first type shows an alphanumeric message with a sentence followed by the number of seats available (see figure 3). Several alphanumeric panels were placed on every floor, at the main entrance and in the centre of every reading/studying room. The second type of information panel uses the floor plan of the library and indicates the availability of seats using LEDs (see figure 4). This type of panel indicates the location and availability of seats with red and green lights. In the library, LED panels are intended to be located at the entrance of each floor.

The design and location of the panels were chosen with a view on maximizing their value to the users of the library. Figure 5 shows the location of the panels. 


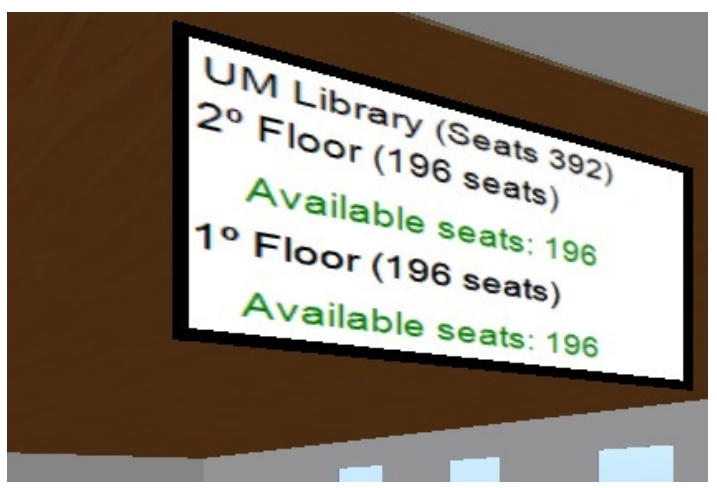

Fig. 3. Alphanumeric panel

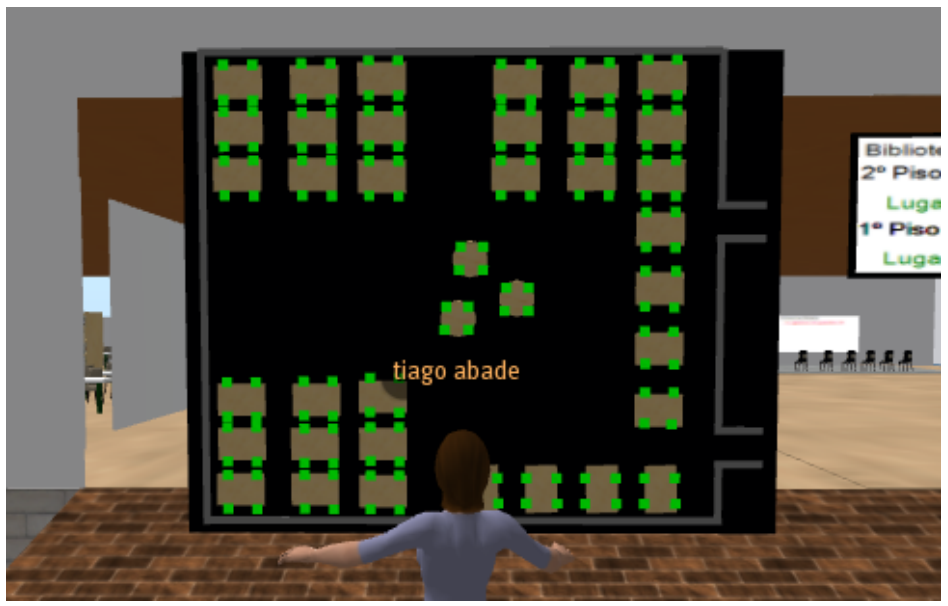

Fig. 4. LED panel

Two types of sensors were used to determine seat availability: pressure sensors and movement sensors. The pressure sensors make it possible to identify occupied seats. The movements sensors, located in the ceiling, can be used to determine the availability of seats by detecting people near the tables.

The design equips chairs with pressure sensors that are triggered by users as they sit down. Every time such an action occurs, this information is sent to every screen present in the building to refresh the data displayed. Movement sensors detect the presence of users when they are close to the seat, and modify the numeric counter when the user gets in or out of their range. The value of the counter is reflected in the screens. 


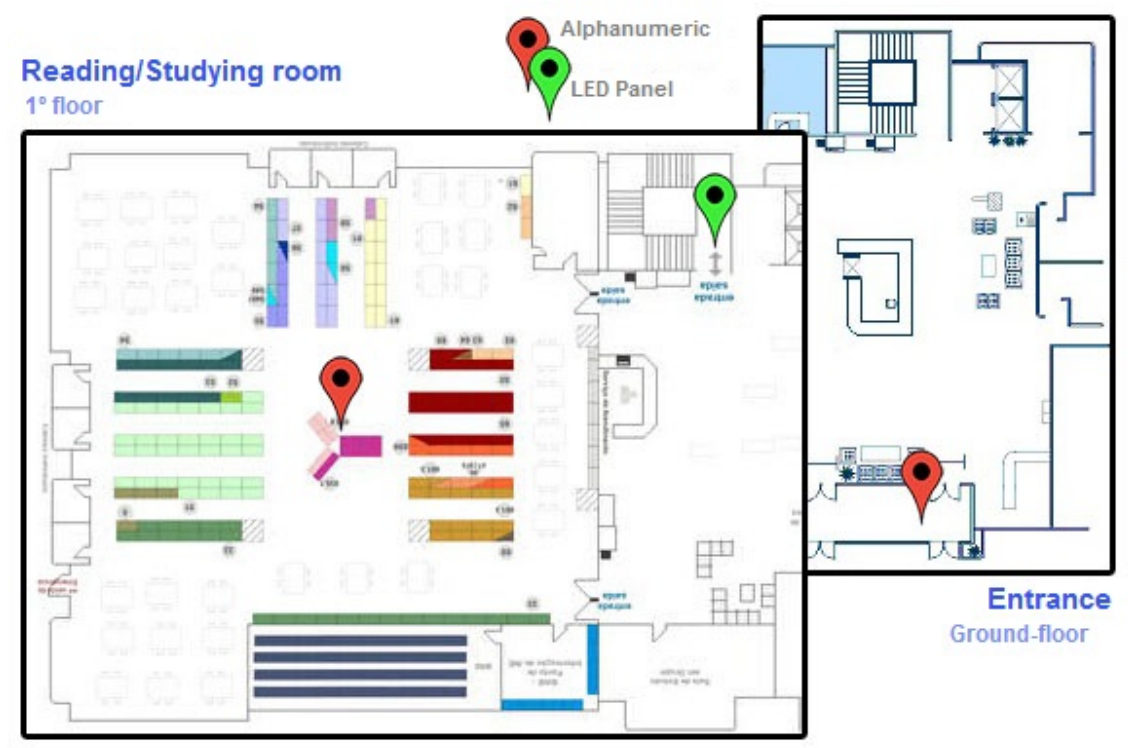

Fig. 5. Painels location

\section{User study}

The user study was designed to evaluate users preferences in relation to the type, location and number of panels. The aim of the study was to predict the utility of the system once fielded. Considering that students are the largest group of users of the library, test subjects were recruited from the student population.

\subsection{Procedure}

The students were briefed with a short introduction about the purpose of the prototype and the information they would find in the simulation. They were asked to enter the library and find an available seat (as they were used to do in the physical library), but this time using the screen panels' assistance.

Each student used the simulation individually but bots were introduced in several seats to improve the realism of the experience. Students were free to explore the environment without being disturbed. No time restrictions were placed in relation to accomplishing the designated task of finding a place to seat.

While in the virtual environment, students were observed, noting their movements and the steps they took to find a place to sit. After completing the tasks, students were asked to answer a questionnaire. The questionnaire was composed of 36 questions, addressing not only the characterisation of the test subjects, and topics related to the screen panels, but also topics related to the use of the virtual environment as a prototype. The questionnaire was divided into the following sections: 


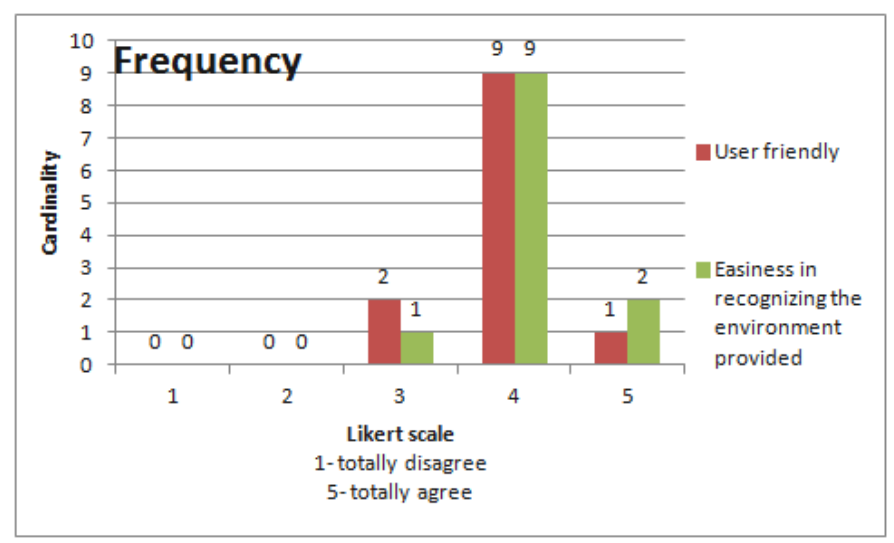

Fig. 6. The prototype as an evaluation artifact

- Profile of the students, including their experience of games using controls similar to those used in this user study.

- Evaluation of the prototype as a tool for evaluation. Here, aspects such as immersion, utility and user satisfaction were measured.

- Evaluation of the prototyped solution, including questions concerned with the location, design and behaviour of the screen panels.

Answers were given in a 5 points Likert scale.

\subsection{Results}

The two main aspects addressed in the study will now be discussed. To simplify presentation, only the more relevant/illustrative questions are addressed.

Twelve students, with an average age of 23 years, participated in the study. Of the 12 students that participated in the study, 10 declared they were familiar with third-person gaming.

The prototype as an evaluation artifact There was positive reaction to the use of a virtual environment as a means of prototyping a ubiquitous system. Students responded positively to whether the environment was easy to use, and whether they were able to recognise the environment that was being evaluated. The mode of responses was 4 (Agree). 9 replies were provided in each case (see Figure 6).

Questions relating to how well the environment supported the identification of alternative solutions, and how effective the environment was in providing an understanding of the design before physical construction, obtained a mode of 5 .

Very high results were obtained for user satisfaction. Whether the approach could be used to validate other environments, and how well this method could be used to explore problems in other areas, obtained modes of 5 (see figure 7). 


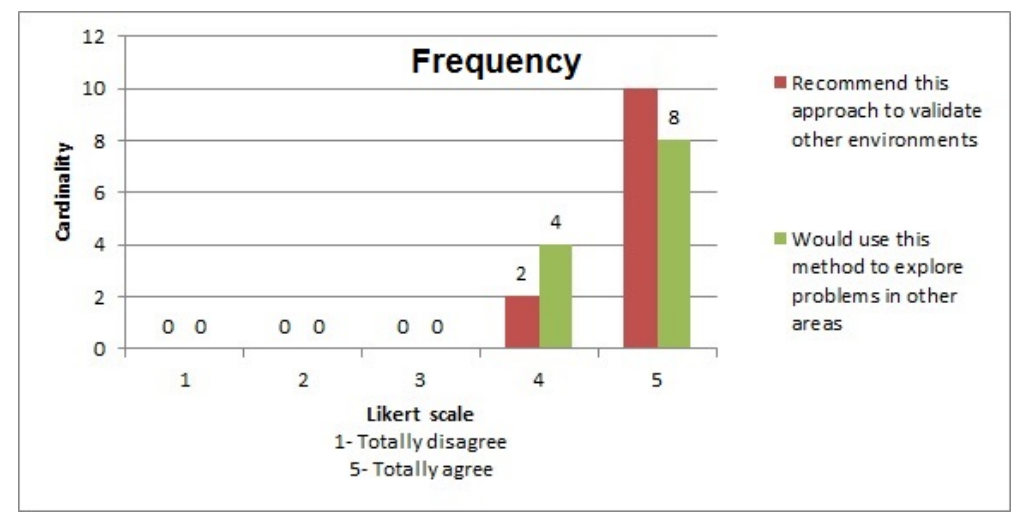

Fig. 7. User satisfaction - frequency graphic

These values demonstrate user satisfaction and a clear understanding of the framework proposed. This is consistent with a previous study where the use of the framework by software engineers was addressed [18].

Screen panels' evaluation All but one of the participants totally agreed with the value of producing a real implementation of the proposed panels in the library on the basis of their evaluation of the simulation. When questioned about the possibility of using the LED panel only, 9 users replied with a mark of 3 or less, clearly showing preference for the joint use of the two types of panels. However, from the observations made during the simulation the participants spent more time watching the LED panel. In reality, during the simulation, in which both panels were available, the tendency of almost every user was to ignore the numeric panel. This suggests that the LED panel might be sufficient, and the preferred source of information.

The location of the panels was another topic addressed in the questionnaire. Here a mode of 3 was obtained regarding the appropriateness of their current location. From the comments collected, and despite several distinct opinions, the most general view was that the panels should be located at the reception on each floor. In addition it was thought that an LED panel should be placed at the centre of each reading/study room so that the students present might be aware of changes in seats availability.

\subsection{Discussion}

The results of the study helped better understand how a system of panels, to help users find available seats in a library, would be used in practice by users of the space. The virtual environment simulation not only helped users get a feel for what the system would be, enabling them to later answer the questionnaire, but also helped us realize that while users were keen to have both types of panels 
present, one of the panels was being more used than the other. This information can be carried forward into making future design decisions. It made it possible to evaluate tradeoffs during design and development.

Besides the information gained regarding the prototyped environment, the fact that users recognised the value of this type of approach to prototyping is also encouraging. The study has some limitations however that must be taken into consideration. Users must be familiar with third person games for example. However the results of users who were not familiar with these games were not substantially different (ease of use and environment recognition had bimodal of 3 and 4 , and 4 and 5, respectively). The fact that there were only two students in this condition means that it is not possible to draw definitive conclusions. This is an aspect that we intend to address in further user studies.

\section{Conclusions}

Experience is difficult to capture as a requirement that could be shown in a system. With the aim to support the design and evaluation of ubicomp environments, a framework for the rapid development of these environments was developed: the APEX framework. This paper describes the application of this framework to a library, exploring the introduction of technology that allows its users to identify available seats. The proposed solution is based on the introduction of sensors and public displays in the library.

A user study was carried out with users of the library. The subjects explored a virtual environment simulation of the proposed smart environment, through avatars who represented them. This approach enabled the evaluation of the environment, but also of the framework itself. The results of the user study indicated the validity of the prototype as a basis for providing early experience to users, helping in the identification of how the system is likely to be used, thus supporting the discovery of aspects that should be improved or modified.

The repetition of these experiments in a CAVE environment, using a 3D stereoscopic projection system, is being carried out. The goal is to check whether immersion has a direct influence on the results. We plan to identify the role of the level of immersion provided by the prototype in the evaluation of ubicomp environments.

As future work, we plan to re-evaluate the location of the panels in the library, considering the avatars' movements in addition to the personal opinions provided. After the redesign, the new results will be compared with the ones reported herein.

\section{Acknowledgments}

The authors wish to thank Michael Harrison for his comments on a previous version of this paper. 
This work is funded by the ERDF - European Regional Development Fund through the COMPETE programme (operational programme for competitiveness) and by National Funds through the FCT - Fundação para a Ciência e a Tecnologia (Portuguese foundation for science and technology) within project FCOMP-01-0124-FEDER-015095.

\section{References}

1. J.J. Barton and V. Vijayaraghavan. UBIWISE, a simulator for ubiquitous computing systems design. Hewlett-Packard Laboratories Palo Alto, HPL-2003-93, 2003.

2. J. C. Campos and M. D. Harrison. Interaction engineering using the ivy tool. In ACM Symposium on Engineering Interactive Computing Systems (EICS 2009), pages 35-44, New York, NY, USA, 2009. ACM.

3. T. Disz and M.E. Papka. UbiWorld: an environment integrating virtual reality, supercomputing, and design. Computing Workshop, pages 46-57, 1997.

4. D. Garlan, D.P. Siewiorek, A Smailagic, and P Steenkiste. Project aura:toward distraction-free pervasive computing. In E. Law, E. Hvannberg, and G. Cockton, editors, Pervasive Computing, pages 22-31. IEEE, 2002.

5. T. Gomes. Master Thesis: 3D Virtual Environments Generation. Master, Universidade do Minho, 2013.

6. B. Hartmann, S.R. Klemmer, M. Bernstein, L. Abdulla, B. Burr, A. RobinsonMosher, and J. Gee. Reflective physical prototyping through integrated design, test, and analysis. In Proceedings of the 19th annual ACM symposium on User interface software and technology, pages 299-308. ACM, 2006.

7. S. Irawati, S. Ahn, J. Kim, and H. Ko. Varu framework: Enabling rapid prototyping of VR, AR and ubiquitous applications. In Virtual Reality Conference, 2008. VR'08. IEEE, pages 201-208. IEEE, 2008.

8. K. Jensen, L.M. Kristensen, and L. Wells. Coloured petri nets and cpn tools for modelling and validation of concurrent systems. In International Journal on Software Tools for Technology Transfer (STTT), chapter 9(3-4), pages 213-254. 2007.

9. Bonnie E. John, Konstantine Prevas, Dario D. Salvucci, and Ken Koedinger. Predictive human performance modeling made easy. In Proceedings of the SIGCHI Conference on Human Factors in Computing Systems, CHI '04, pages 455-462. ACM, 2004.

10. Yang Li and J.I. Hong. Topiary: a tool for prototyping location-enhanced applications. Proceedings of the 17th annual ACM, 6(2):217-226, 2004.

11. S.A. Nazari and A. Klar. 3DSim: Rapid Prototyping Ambient Intelligence. In SOc-EUSAI conference, pages 303-307. ACM, 2005.

12. H. Nishikawa, S. Yamamoto, M. Tamai, K. Nishigaki, T. Kitani, N. Shibata, K. Yasumoto, and M. Ito. UbiREAL: Realistic smartspace simulator for systematic testing. Lecture Notes in Computer Science, 4206/2006:459-476, 2006.

13. E. O'Neill, D. Lewis, and O. Conlan. A simulation-based approach to highly iterative prototyping of ubiquitous computing systems. In 2nd International Conference on Simulation Tools and Techniques, pages 56-66. ICST (Institute for Computer Sciences, Social-Informatics and Telecommunications Engineering), 2009.

14. J. L. Silva. PhD Thesis. Rapid prototyping of ubiquitous computing environments. $\mathrm{PhD}$ thesis, Universidade do Minho, 2012. 
15. J. L. Silva, J. C. Campos, and M. D. Harrison. An infrastructure for experience centered agile prototyping of ambient intelligence. In symposium on Engineering interactive computing systems, pages 79-84. ACM SIGCHI, 2009.

16. J. L. Silva, J. C. Campos, and M. D. Harrison. Formal analysis of ubiquitous computing environments through the apex framework. In Symposium on Engineering interactive computing systems, pages 131-140. ACM SIGCHI, 2012.

17. J. L. Silva, Ó. Ribeiro, J. Fernandes, J. Campos, and M. Harrison. The apex framework: prototyping of ubiquitous environments based on petri nets. In HumanCentred Software Engineering. Lecture Notes in Computer Science, chapter 6409, pages 6-21. Springer, 2010.

18. J.L. Silva, J.C. Campos, and M.D Harrison. Prototyping and analysing ubiquitous computing environments using multiple layers. International Journal of HumanComputer Studies. accepted. 